\title{
Microsymposium
}

MS23.006

\section{Operando studies of nanoscale olivine cathodes for Li-ion batteries}

\author{
D. Ravnsbaek ${ }^{1}$, K. Xiang ${ }^{1}$, W. Xing ${ }^{1}$, P. Gionet ${ }^{2}$, Y. Chiang ${ }^{1}$, P. Chupas ${ }^{2}$, K. Chapman ${ }^{2}$, Y. Chiang ${ }^{1}$ \\ ${ }^{1}$ MIT, Department of Material Science and Engineering, Cambridge, USA, ${ }^{2}$ A123-Systems, Waltham, USA
}

Compounds of interest for ion storage in advanced batteries frequently exhibit phase transformations, driven by large and variable electrochemical driving forces inherent to practical use. Understanding how materials variables (e.g. composition, nanoscalecrystallite size and dynamic electrochemical conditions) affect the phase transition is of vital importance for practical applications as the reversibility and stability of these structural transformations determine the energy, power, and lifetime of the system. Due to its outstanding power, safety and cycle-life olivine LiFePO4 (LFP) has during the past decade become a widely used, and one of the most well-studied, lithium ion battery cathode materials. It is well-established that for LiFePO4 the storage/release of lithium is accompanied by a first-order phase transition between lithiated and delithiated states. However, it would be a mistake to conclude that the behavior of pure LFP is representative of all olivines, in particular the vast range of doped and mixed-metal olivines that are also of interest for their advantageous electrochemical properties.1,2 Utilizing operando synchrotron radiation powder X-ray diffraction (SR-PXD), we demonstrate here, by systematic screening of the electrochemical driven phase transitions in a series of LiMnyFe1-yPO4 ( $y=0.1-0.8$ ) powders, a completely different phase transformation mode dominated by formation of metastable solid solutions for nanoscale LMFP compared to the binary lithiation states within the extremely well-studied case of LFP. Through Rietveld refinement the misfit strains during phase transformations are examined, revealing small elastic misfits between phases within the extended solid solution regime. On the basis of the time- and state-of-charge dependence of the olivine structure parameters, we propose a coherent transformation mechanism, and finally, we bring evidence that the observed metastability is enabled by particle size reduction to the nanoscale.

[1] Meethong et al. Adv. Funct. Mater. 2009,19,1060., [2] Chung et al. Nat. Mater. 2002,1,123.
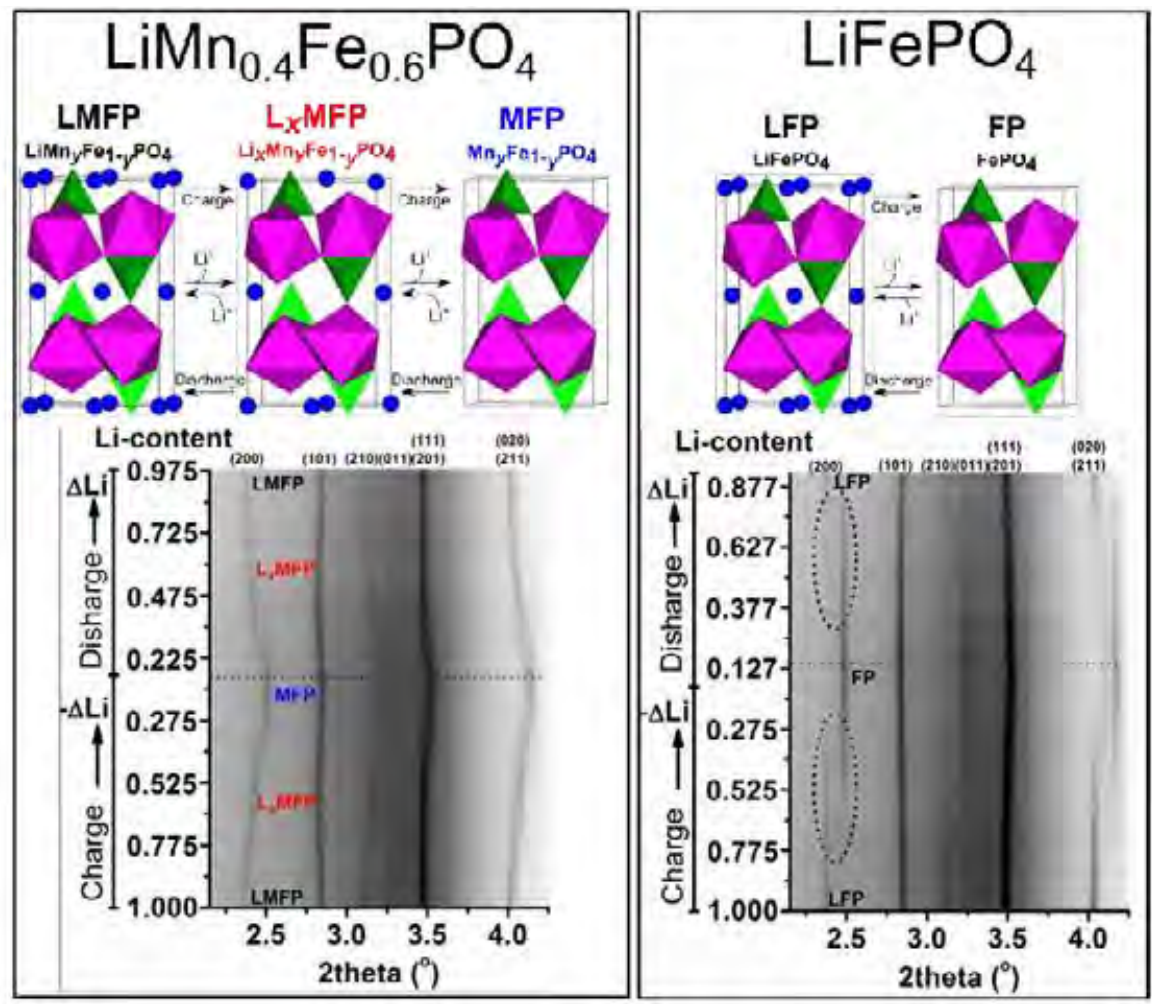

Keywords: Operando, Li-ion batteries, Phase transformations 